\title{
Dynamics of multilayer networks with amplification
}

Thierry Njougouo, ${ }_{1}^{1}$ Victor Camargo, ${ }^{2,3}$ Patrick Louodop, ${ }^{1}$ Fernando Fagundes Ferreira, ${ }^{2,3}$ Pierre K. Talla, ${ }^{4}$ and Hilda A. Cerdeira ${ }^{5}$

1) Research Unit Condensed Matter, Electronics and Signal Processing, University of Dschang, P.O. Box 67 Dschang,

Cameroon.

${ }^{2)}$ Center for Interdisciplinary Research on Complex Systems, University of Sao Paulo, Av. Arlindo Bettio 1000, 03828-000 São Paulo, Brazil.

${ }^{3)}$ Department of Physics-FFCLRP, University of São Paulo, Ribeirao Preto-SP, 14040-901,

Brasil.

4) L2MSP, University of Dschang, P.O. Box 67 Dschang, Cameroon.

${ }^{5)}$ São Paulo State University (UNESP), Instituto de Física Teórica, Rua Dr. Bento Teobaldo Ferraz 271, Bloco II, Barra Funda, 01140-070 São Paulo, Brazil.

(Dated: 4 December 2020)

We study the dynamics of a multilayer network of chaotic oscillators subject to an amplification. Previous studies have proven that multilayer networks present phenomena such as synchronization, cluster and chimera states. Here we consider a network with two layers of Rössler chaotic oscillators as well as applications to multilayer networks of chaotic jerk and Liénard oscillators. Intra-layer coupling is considered to be all to all in the case of Rössler oscillators, a ring for jerk oscillators and global mean field coupling in the case of Liénard, the inter-layer coupling is unidirectional in all these three cases. The second layer has an amplification coefficient. An in-depth study on the case of a network of Rössler oscillators using master stability function and order parameter leads to several phenomena such as complete synchronization, generalized, cluster and phase synchronization with amplification. For the case of Rössler oscillators, we note that there are also certain values of coupling parameters and amplification where the synchronization doesn't exist or the synchronization can exist but without amplification. Using other systems with different topologies, we obtain some interesting results such as chimera state with amplification, cluster state with amplification and complete synchronization with amplification.

Research on multilayer networks has attracted a lot of attention in recent years in many areas of physics, engeneering, social sciences et ${ }^{1+6}+6$. Some emergent behaviors in such systems due to interaction among the dynamical units reveal a variety of interesting phenomena, such as synchronization ${ }^{1778}$, cluster formation ${ }^{9}$, explosive synchronization ${ }^{10}$, explosive desynchronization ${ }^{11}$, chimera $^{12-16}$ etc. Among these, synchronization and chimeras are the most widely studied. The notion of amplification is very important in science and technology. This work presents an investigation of different phenomena such as complete synchronization, cluster formation, phase synchronization and chimera states in a network with amplification. For an extended study we present three cases with three different topologies.

\section{INTRODUCTION}

The structure of many real world problems in nature, engineering, science and technology is defined as a set of entities interacting with each other in complicated patterns that can produce multiple types of relationships which change in time and exhibits a plethora of emergent patterns or behaviors as synchronization, chimeras, chaos, consensus, cooperation, only to mention a few. One very important ingredient behind most of those phenomena is the way in which the particles or agents forming the systems interact, i.e., the topology of the underlying network.

Network theory is used as an important tool for the mod- elling of dynamical processes in complex systems ${ }^{1227}$. It plays also a major role in the investigation of collective behavior. It finds many applications in epidemiology where it is used to investigate epidemic spreading, in industry where it is used in control of behavior of machines, in dynamics of populations with the control of displacement of the individuals, the cars, the drones or the airplanes. According to the these applications, we can mention that the main objective is the controllability of the network to lead to a certain state (it can be synchronization, cluster state, phase-flip, chimera state, etc. $)^{8 / 13 / 14] 17-19}$. Thus, the investigation of the dynamics of the networks need the expertise of some mathematical tools such as the Master Stability Function (MSF) developed by Pecora and Carroll, the transversal Lyapunov exponent, the correlation between the oscillators of the same or of a different layer, etc. One of the best methods to study the stability of the synchronization in the network is the Master Stability Function 20 . This method is used for coupled identical oscillators.

Many researchers are studying several phenomena which take place in multiplex networks. Such interest is motivated in understanding how the complete or partial synchronization occurs in this type of systems and also because the topologies of multiplex networks appear in several natural and tecnological systems. Multiplex network may be described as being a collection of two or more coupled networks where a set of networks is connected by links where the interactions are of different types ${ }^{21 / 22}$. These links characterize the connections existing between any node or network of the multiplex network. Many recent works addressing multilayer structures and systems were summarized in 23 .

The study of inter-layer synchronization in non-identical 
multilayer networks was address in 24 . The authors were able to show an analytical treatment for a two-layer multiplex using the Master Stability Function method. One interesting outcome was to predict the effect that missing links in one of the layers has on the inter-layer synchronization. Later, in ${ }^{25}$ it was found that a sparse inhomogeneous second layer can promote chimera states in a sparse homogeneous first layer. The study of synchronization of non-identical multilayers is very recent, thus many collective behavior properties and patterns may unravel.

Here, we consider a network with two layers where we choose an all to all coupling in the layer for the case of Rössler oscillators, a ring with bidirectional coupling in each layer for the case of jerk oscillators and aglobal mean field coupling for the last case mentioned above. The connection between the systems of both layers (interlayer coupling) is unidirectional. The main goal of this work is to investigate in each case the dynamics of each layer as well as the whole network with amplification in the second layer. We found the key values of the parameters to control synchronization with and without amplification.

The remainder of this work is organized as follows. In section II, we present our multilayer network with the mathematical description of the model and the systems. The dynamics of the main case is presented in section III emphasizing on the intralayer and interlayer coupling in which numerical simulations are done. An application to another two systems has been studied in section IV and finally, we present the conclusions in section V.

\section{MULTILAYER NETWORK}

The model consists in a multilayer network constituted of $N$ nodes connected in each layer, which can be represented by a $2 N \times 2 N$ adjacency matrix $A_{i j}$ where the elements of this matrix are respectively 1 if the nodes $i$ and $j$ are connected and 0 if not. Based on Ref. ${ }^{25}$, the adjacency matrix of the whole network consisting two layers can be expressed as follow:

$$
\mathbf{A}=\left(\begin{array}{cc}
A^{1} & 0 \\
I & A^{2}
\end{array}\right)
$$

where $A^{1}$ and $A^{2}$ are the $N \times N$ adjacency matrix modelling the intralayer connectivity in the first and second layer respectively. $I$ is an $N \times N$ identity matrix representing the unidirectionnal interactions (Layer 1 -> Layer 2) between the oscillators with the same index in both layers. The use of the null matrix is justified by the non-existence of a connection from the slave layer to the master layer. In the following, we consider a model of multilayer network constituted of $N$ nodes in each layer connected using an all-to-all coupling scheme in each layer (see Fig 11). To each node corresponds a nonlinear autonomous Rössler oscillator as described in 26. Notice that it is this combined oscillator which defines our network as a two-layer system made up of a driving system and a slave one.

The dynamics of the first layer also considered as the driver for the network is described by $\mathrm{Eq} 2$ where $\varepsilon_{1}$ is the

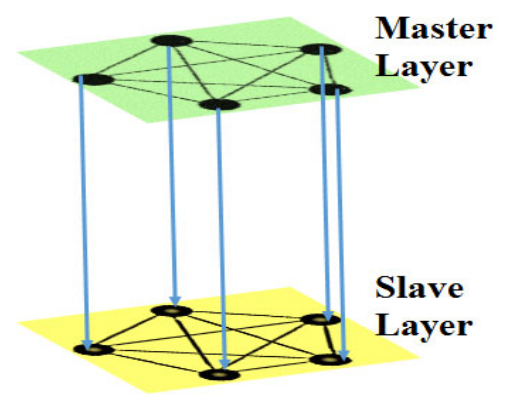

FIG. 1: Schematic representation of a network with two layers of interaction.

intra-layer coupling strength for the first layer.

$$
\left\{\begin{array}{l}
\dot{x}_{i}^{1}=-x_{i}^{2}-x_{i}^{3}+\varepsilon_{1} \sum_{k=1}^{N} A_{i k}^{1}\left(x_{k}^{1}-x_{i}^{1}\right), \\
\dot{x}_{i}^{2}=x_{i}^{1}+a x_{i}^{2} \\
\dot{x}_{i}^{3}=b x_{i}^{1}+x_{i}^{3}\left(x_{i}^{1}-c\right) .
\end{array}\right.
$$

The second layer has exactly the same intra-layer topology with similar systems in the nodes. The choice of systems in the second layer follows Louodop et al. ${ }^{[2]}$ where the authors show in Appendix A that, this form of coupling between elements of different layers produces generalized synchronization 27. Notice that it is this system defined in Louodop et al[27, what defines which oscillators in Layer 2 interact with those in Layer one, while keeping the same all to all intra-layer topology. Therefore, the dynamics of the second or slave layer is given by $\mathrm{Eq} 3$.

$$
\left\{\begin{array}{l}
\dot{y}_{j}^{1}=-y_{j}^{2}-y_{j}^{3}+\varepsilon_{2} \sum_{k=1}^{N} A_{j k}^{2}\left(y_{k}^{1}-y_{j}^{1}\right)+C_{0}\left(x_{j}^{1}-C_{2} y_{j}^{1}\right), \\
\dot{y}_{j}^{2}=\frac{x_{j}^{1}+a x_{j}^{2}}{C_{2}} \\
\dot{y}_{j}^{3}=b y_{j}^{1}+\frac{x_{j}^{3} x_{j}^{1}}{C_{2}}-c y_{j}^{3} .
\end{array}\right.
$$

Here, $\varepsilon_{2}$ is the intra-layer coupling strength and $C_{0}$ is the interlayer coupling strength. It is important to mention that, this inter-layer coupling exists only between the oscillators with the same index (i.e. for $i=j$ where the $j$ index runs from 1 to $N) . C_{2}$ is the parameter of proportionality named amplification coefficient. This term or interaction via a conjugate variable has been used in the literature to model revival 2829 as well as amplitude death $h^{30}$. For all these layers we consider $a=0.36, b=0.4$ and $c=4.5$ and we note that at these values of the parameters the systems operate in the chaotic regime ${ }^{26}$. This topology of connectivity between the master and the slave layer imposes generalized synchronization between both layers in the absence of intralayer coupling because as it is conceived (see Appendix A), the slave layer is supposed to function as an observer of the master layer with certain conditions $C_{2} \neq 0$. In this work, we were interested 
in the notion of synchronization with amplification (or reduction) depending on the value of $C_{2}$. For $C_{2}>1$ we have an amplification of the systems of the master layer (or a reduction of the systems of the slave layer) and conversely for $C_{2}<1$. It should be noted that when $C_{2}$ takes negative values there is an anti-synchronization between the systems of the master layer and those of the slave layer having the same index. The amplification coefficient must be different from zero $\left(C_{2} \neq 0\right)$ and bounded, because if $C_{2}=0$ the systems of the slave layer will diverge and if $C_{2}$ is too large the slaving factor will tend to zero. Therefore we keep $C_{2}$ in the interval between 0.005 and 2. This type of topology finds applications in many domains such as aircraft control where recently they have proven that an optimal control permitted to regulate the air traffic in the sky 31. Considering the domains of application of this network topology, it seems rather important to investigate the dynamics of this network with different parameters, which is presented in the next section.

\section{DYNAMICS OF NETWORKS}

\section{A. Dynamics of Different Layers}

Considering the topology given in Fig 1 and the mathematical equations for both layers $\mathrm{Eq} 2$ and $\mathrm{Eq} 3$ respectively, we are going to investigate numerically the dynamics in the different layers using the MSF described in Appendix B. A numerical calculation is done using Runge-Kutta fourth order for a long time simulation and the permanent solutions are considered at $t_{\min }=0.6 t_{\max }$.

To illustrate the behaviour of the oscillators of the first layer we present in Fig 2(a) the Master Stability Function or Largest Lyapunov Exponent ( $L L E$ ) of the variational equation Eq. B10 used to characterize the stability of the synchronization in the first layer. In this figure there are two important regions in terms of characterization of the stability of the synchronization: if the $L L E \leq 0$ the synchronization is stable and the $L L E>0$ the synchronization is unstable. By varying smoothly the intralayer coupling $\varepsilon_{1}$, this figure shows that, when $\varepsilon_{1}$ increases the systems evolve to the synchronous state at $\varepsilon_{1}=0.009$. This synchronization is obtained at a minimal value of the coupling strength in the first layer and we note that the synchronization in the slave layer is highly influenced not only by the coupling but also by the amplification, this can be seen on the behavior of the order paramete ${ }^{\sqrt{32 / 33}}$ which is plotted in Fig 2 b) as a function of $\varepsilon_{1}$. The procedure to compute the order parameter is explained in Appendix C. It should be noted that the notion of amplification here is related to the amplitude of the oscillations of the state variables of the systems. We are talking about synchronization with amplification if and only if the ratio $X / Y=C_{2}$ is respected, as shown in Appendix A. So, we investigate here the impact of the amplification parameter on the dynamics of the network, where the order parameter of the slave layer is plotted for different values of $C_{2}$. According to this figure although interaction between the layers does not impede synchronization in the slave layer, it becomes more effective for small values of $C_{2}$. Based on the demonstration given in Appendix A synchronization occurs at $Y=\frac{X}{C_{2}}$. Therefore, when $C_{2}<1$ we obtain an amplification in the slave layer and respectively a reduction in master layer and vice-versa when $C_{2}>1$. Thus, if we need to achieve synchronization in both layers at the same value of the interlayer coupling, $C_{2}$ must be very small, leading to a significant amplification at the second layer. Also from Fig 2 b) we see that for the minimum value of $C_{2}=0.005$, the synchronization in both layers happens at the corresponding values of $\varepsilon_{1}$ and $\varepsilon_{2}$ $\left(\varepsilon_{2}=10 \varepsilon_{1}\right)$, this is represented in Fig 2 (b), where the order parameter $r_{1}$ and $r_{2}$ for both layers is seen to reach the value for complete synchronization at the same point. This value of $C_{2}$ produces an amplification of around 200 of all the variables of the master layer $(Y \approx 200 X)$ in the slave layer. In Fig, $2, \mathrm{c})$ we plot the mean phase of the driver and the slave layer (the first 30 systems are for the driver layer and the rest for the slave layer) for the value $\varepsilon_{1}=0.005$ of the intralayer coupling. The situation shown here is confirmed by the Fig 2 a) $(L L E>0)$. If we consider $\varepsilon_{1}=0.007$, Fig 2a) shows that the Largest Lyapunov Exponent is non negative but very close to zero, then in the Fig 2(d) we show the mean phase where the master layer has a two cluster synchronization with equal phases 91034 . In the slave layer, while the clusters follow the systems with the same index as that of the master layer, we see an oblique sliding of the systems reminding of a splay state. To better appreciate the dynamics of the oscillators in this behaviour, we show in Fig 2 (g) the attractors of the oscillators labeled $1,6,11,16,21,26$ in the master and slave layers for $C_{2}=2$ and $\varepsilon_{1}=0.007$ ( $L L E \geq 0$ ). For $\varepsilon_{1}=0.009$ (with $L L E<0$ ), we obtain Fig 2(e) which represents the synchronization in the first layer and a coherent oblique sliding in the second layer. By computing the phase difference between consecutive oscillators (here consecutive refers to the indices of the oscillators) we verified that the phase distance between oscillators of consecutive index in the slave layer is constant, therefore the second layer presents indeed a phenomenon of splay $37 / 38$. In Fig 2(f) we show for $\varepsilon_{1}=0.04$ the phase synchronization in both layers but not at the same value of the mean phase. So, for the multilayer network, the dynamics is equivalent to that of two clusters. To illustrate the dynamics of the system we show in Fig $2(\mathrm{~h})$ the attractors of some oscillators (labeled $1,6,11,16,21,26)$ for $\varepsilon_{1}=0.04$ to appreciate the behaviour of the entire network.

Therefore, we can conclude that, according to the different values of the intralayer coupling the network leads to different phenomena such as, cluster synchronization, splay, synchronization and the stability of this synchronization is confirmed by the MSF.

\section{B. Impact of the interlayer coupling and amplification on the dynamics of the network}

In the previous Sections we have shown the influence of the intralayer coupling on the dynamics of the network. We see that the network presents many phenomena depending on the amplification coefficient $C_{2}$ and the interlayer coupling $C_{0}$. In this section, our goal is twofold: first we investigate the 

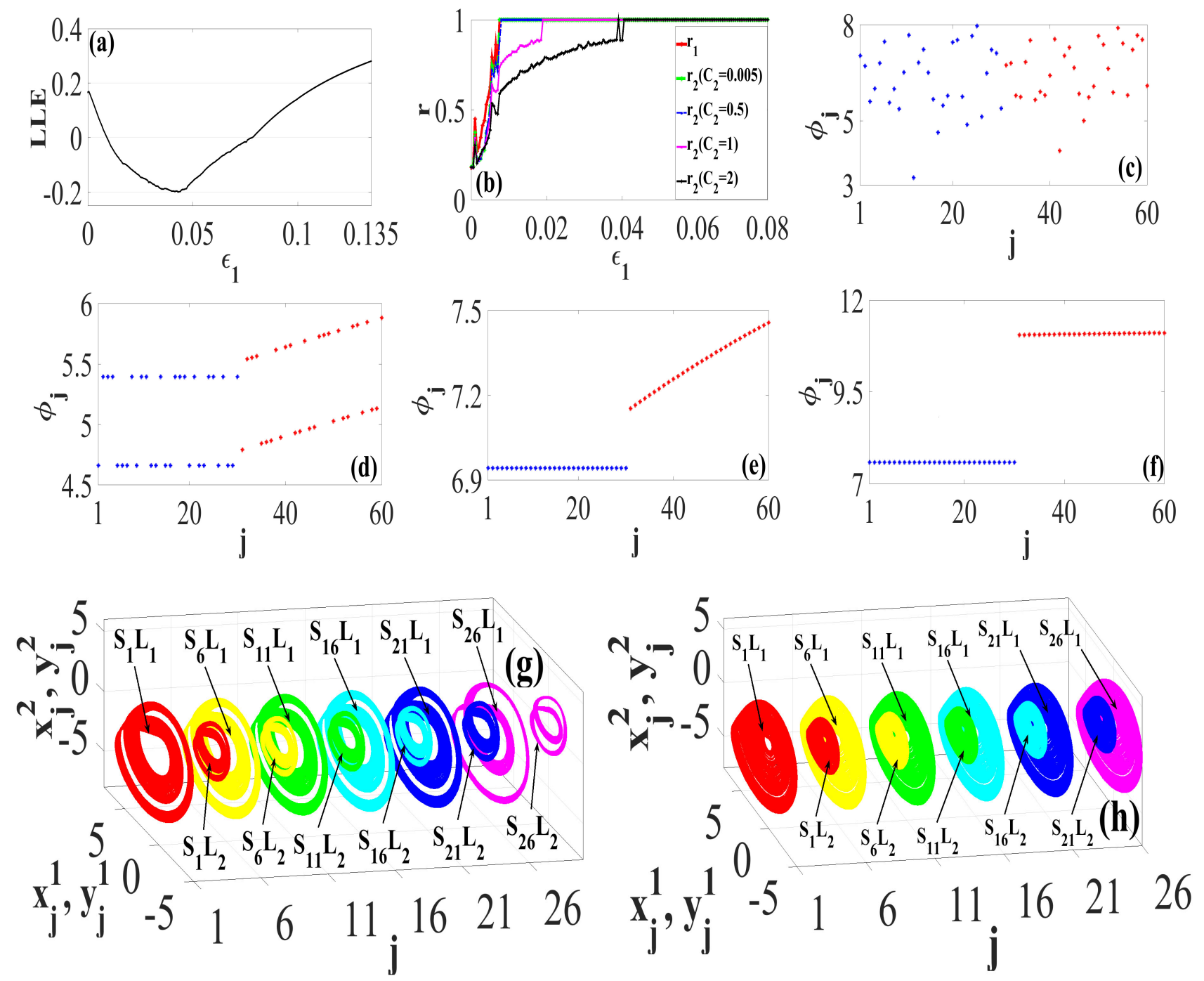

FIG. 2: Dynamics of the two layers of the network: (a) Master stability function of the first layer as a function of the intralayer coupling considering $\varepsilon_{2}=10 \varepsilon_{1}, C_{0}=1$ and $C_{2}=2$. (b) Order parameter showing the dynamics of the slave layer for different values of the amplification coefficient for $\varepsilon_{2}=10 \varepsilon_{1}$ and $C_{0}=1$. (c,d,e and f) Mean phase of the first and second layer respectively for $\varepsilon_{1}=0.005,0.007,0.009,0.04, C_{0}=1$ and $C_{2}=2$. (g) Attractors of the oscillators 1, 6, 11, 16, 21 and 26 in the master and slave layer for $\varepsilon_{1}=0.007$. (h) Attractors of the oscillators 1, 6, 11, 16, 21 and 26 in the master and slave layer $\varepsilon_{1}=0.009$ respectively. $S_{i} L_{l}$ means system $i$ of the layer $l(i=1,2, \ldots, N$ and $l=1,2)$.

behaviour of the network under the impact of these two parameters and then we show the effect of the amplification as well as its effectiveness in the network. We keep the intracoupling constants $\left(\varepsilon_{1}, \varepsilon_{2}\right)$ fixed, varying smoothly the amplification coefficient $\left(C_{2}\right)$ from 0.005 to 2 and the inter-coupling $\left(C_{0}\right)$ from 0 to 20.

As mentioned in Fig 2(b), the synchronization in the slave layer is imposed by the synchronization in the master layer. According to the literature ${ }^{39140}$, in order to bring our multilayer network towards a desired behaviour such as synchronization, cluster formation, splay and so on, it suffices to adjust the coupling. Although this is usually the case, in our system we have two important parameters acting as interlayer couplings $\left(C_{0}\right.$ and $\left.C_{2}\right)$ with the difference that one of them $\left(C_{2}\right)$ allows us to increase or decrease the amplitude of the sig- nal in one of these layers. To illustrate the evolution towards the synchronization as a function of the amplification parameter $C_{2}$ and the interlayer coupling $C_{0}$ we show in Fig 3 (a) the order parameter of the slave layer, since we are interested in synchronization throughout the network. By considering the intralayer coupling $\varepsilon_{1}=0.03$ and $\varepsilon_{2}=0.25$ all the oscillators of the first layer are synchronized but due to the effects of the amplification parameter $C_{2}$ and the interlayer coupling, the dynamics of the slave layer can not be the same. Therefore, the dynamics of the multilayer network and particularly the slave layer is greatly affected by $C_{0}$ and $C_{2}$. This can be observed in Fig 3 (a) where we plot the order parameter of the second layer. In red we represent the region where synchronization and amplification is obtained. We note that in this red zone the relation $X=C_{2} Y$ is verified with a precision of 

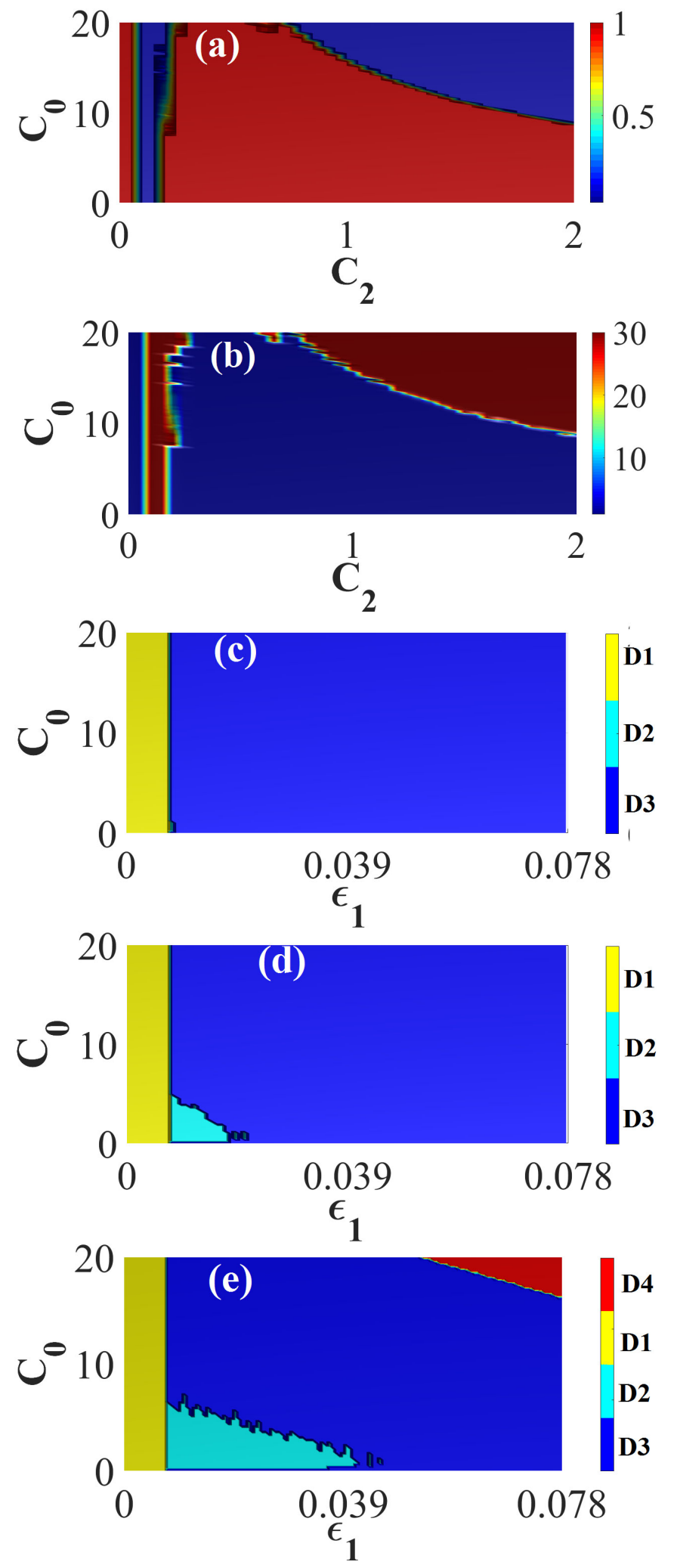

FIG. 3: Network synchronization regions: (a) Order parameter of the multilayer network for $\varepsilon_{1}=0.03$ and $\varepsilon_{2}=0.25$ as a function $C_{0}$ and $C_{2}$. (b) Number of states in the slave layer for $\varepsilon_{1}=0.03$ and $\varepsilon_{2}=0.25$ as function $C_{0}$ and $C_{2}$. Two parameter phase diagram by simultaneously varying the intra-layer coupling $\varepsilon_{1}$ and inter-layer coupling $C_{0}$ with $\varepsilon_{2}=10 \varepsilon_{1}$ and for different values of the amplification coefficient $C_{2}$ : (c) $C_{2}=0.5$, (d) $C_{2}=1$ and (e) $C_{2}=2$.
$10^{-4}$, while in the blue domain it is possible to obtain synchronization in the first layer only but not in both layers of the network. This information is corroborated by the number of states of the second layer shown in Fig 3 (b). This Fig 3 (b) confirms the order parameter by presenting a number of states equal to one in the case of synchronisation (dark blue). When the order parameter is different from one, the synchronisation of all the systems in the network is not achieved. At the moment, we can find partial synchronization called cluster which is characterized by a number of independent states of the network much less than the total number of elements. Based on Fig 3 (b), we see a thin region with cluster formation in the area of transition from synchronization to desynchronization and vice versa. To better highlight the impact of the amplification $\left(C_{2}\right)$ on the transition to synchronization, we have presented the order parameter of the multilayer network showing the transition to the synchronous state for three values of the amplification $C_{2}=0.5, C_{2}=1$ and $C_{2}=2$, showed in Fig 3 (c, d and e) respectively. Varying smoothly the intra and interlayer coupling for these three fixed values of $C_{2}$, we have obtained different areas of synchronization of the systems of the network. For these three figures the first domain (D1) represents the region where there is no synchronization in any layer and the second domain (D2) where the synchronization exists only in the first layer. The third domain (D3) represents the zone of synchronization of both layers and the last domain (D4) is showing the area where there is divergence between the states of the oscillators of the slave layer. Here, divergence means an infinite amplification of the amplitude of the oscillations of the systems of the slave layer leading to an explosion $\left(y^{1}, y^{2}, y^{3}\right.$ tend towards the inifinite). In summary, we notice that, the area of synchronization of both layers increases when the amplification coefficient decreases. So in Fig 3 (c) this zone of synchronization of both layers is the largest domain (D3) and the zone (D2) where only the master layer synchronizes is almost nonexistent. Therefore when $C_{2}$ increases, it takes stronger values of the coupling in the master layer for the slave layer to become synchronized as can be seen in Fig 2(b). Fig 4 is used to confirm and to present the transition to the synchronization using the Pearson correlation ${ }^{41}$ (defined by Eq 4 ) between the first variables of each oscillator of both layers, the time series of the first variables of the oscillators label 1 and 15 of both layers and the mean phase of the oscillators of the network.

$$
\rho\left(x^{1}, y^{1}\right)=\frac{\sum_{i=1}^{N}\left(x_{i}^{1}-\bar{x}^{1}\right)\left(y_{i}^{1}-\bar{y}^{1}\right)}{\sqrt{\sum_{i=1}^{N}\left(x_{i}^{1}-\bar{x}^{1}\right)^{2}} \sqrt{\sum_{i=1}^{N}\left(y_{i}^{1}-\bar{y}^{1}\right)^{2}}}
$$

where $\bar{x}^{1}=\sum_{i=1}^{N} x_{i}^{1}$ and $\bar{y}^{1}=\sum_{i=1}^{N} y_{i}^{1}$ are the mean of the states variables $x_{i}^{1}$ and $y_{i}^{1}$. In this manuscript, the colour yellow used in the correlation refers to the oscillators that synchronize and the colour blue to those that do not synchronize.

Fig 4 (a) shows the correlation between the first variables of the oscillators of the master and slave layer for $C_{0}=10$ and 

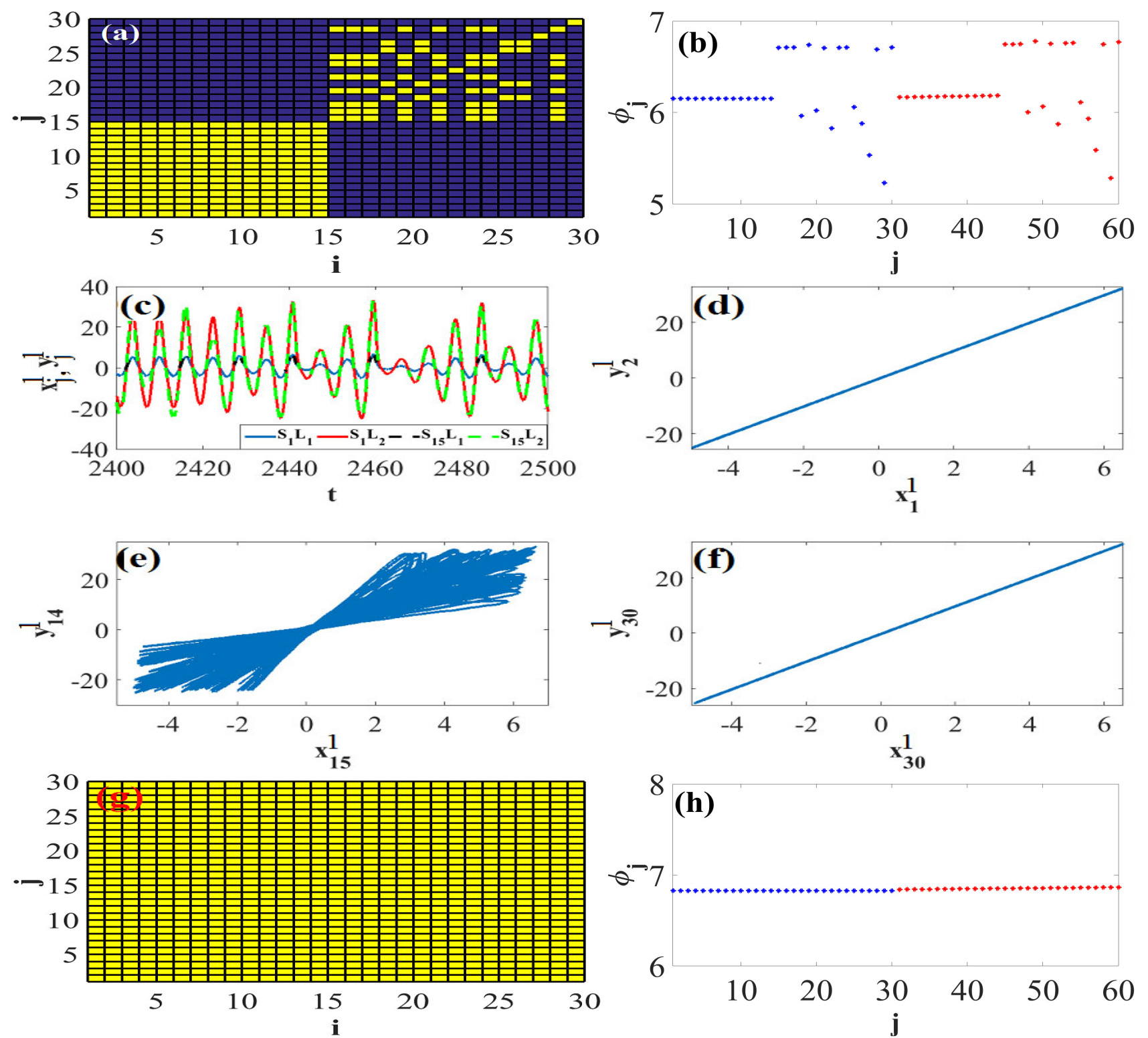

8 (h)
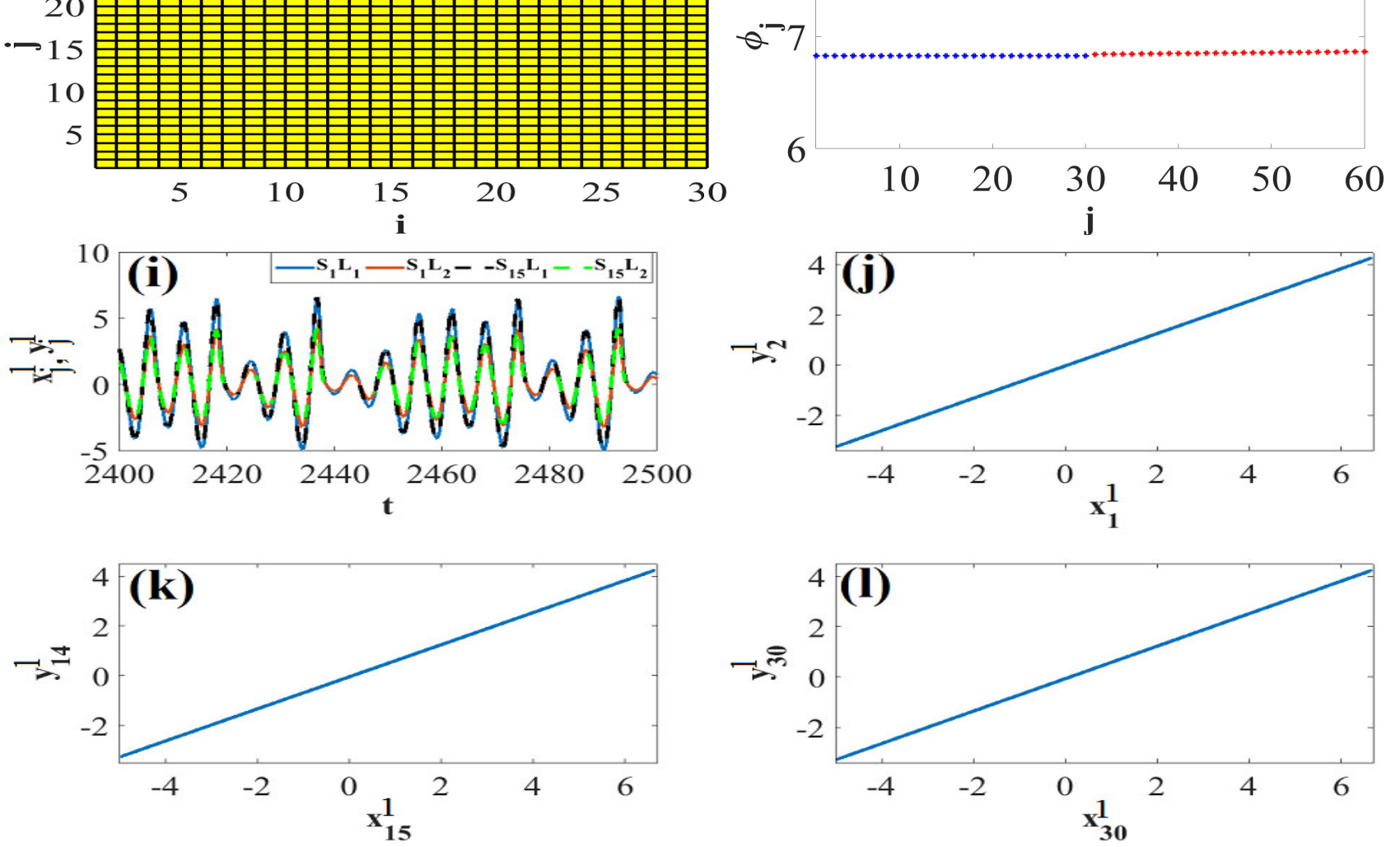

FIG. 4: Dynamics of the multi-layer network for $C_{0}$ and $C_{2}$ : (a) Correlation between $x_{i}^{1}$ and $y_{j}^{1}$ (Yellow color indicates the oscillators in the synchronized state and blue the oscillators in an unsynchronized state.) (b) Mean phase and (c) time series of some oscillators of the network for $C_{2}=0.2$ and $C_{0}=10 . S_{i} L_{l}$ means system $i$ of the layer $l(\mathrm{i}=1,2, \ldots, \mathrm{N}$ and $1=1,2)$. (d,e,f) Synchronization between some oscillators in the first and second layer of the network for $C_{2}=0.2$ and $C_{0}=10$. (g) Correlation between $x_{i}^{1}$ and $y_{j}^{1}$. (h) Mean phase and (i) time series of some oscillators of the network for $C_{2}=1.55$ and $C_{0}=10$. $(\mathrm{j}, \mathrm{k}, \mathrm{l})$ Synchronization between some oscillators in the first and second layer of the network for $C_{2}=1.55$ and $C_{0}=10$. Figures $(\mathrm{d}, \mathrm{e}, \mathrm{f})$ and $(\mathrm{j}, \mathrm{k}, \mathrm{l})$ are special cases of the correlations presented in Figures 4(b and g). However, these figures even if they may confuse for being similar show that amplification clearly

depends on the value of $C_{2}$, and also show that in some cases the synchronisation of oscillators of different index is possible (see Figs. $4 \mathrm{~d}$ and $4 \mathrm{k}$ ). 
$C_{2}=0.2$ (these values are taken almost at the border of the separation of the red and blue zone in Fig.3(a)). This figure presents yellow and blue colors to identify those oscillators of the multilayer network that synchronize and those who do not synchronize, respectively. In this figure, the $\mathrm{x}$-axis corresponds to the index used to identify the oscillators of the master layer $(i)$ and the y-axis corresponds to the index used to identify the different oscillators of the slave layer $(j)$. According to this figure, the first 14 oscillators of both layers synchronize. To present in detail the situation we plot in Fig 4 (b) for the same fixed values of the parameters the mean phase for oscillators in both layers, where the labels 1 to 30 belong to the master layer and 30 to 60 to the slave layer. Here we notice two different groups in each layer: the synchronous group (first 14 oscillators of each layer) and the asynchronous group, so the transition to the synchronization is done by cluster formation. To show the effect of the amplification for these fixed values of parameters, we have in Fig 4 (c) the time series of the variables for oscillators labels 1,and 15. This figure presents a chaotic evolution of these variables as a function of time as well as the amplification, these effects of synchronization with amplification are well appreciated in Fig $4(d, f)$ for the oscillators indicated in the axes. According to Fig 4 (a) the $15^{\text {th }}$ oscillator of the master layer and the $14^{\text {th }}$ oscillator of the slave layer can not synchronize (see Fig 4(e)). At the end, to confirm the synchronization with amplification in the multilayer network, we present in Fig $4 \mathrm{~kg}$ ) the correlation between the elements of both layers for $C_{0}=10$ and $C_{2}=1.55$. This correlation shows the synchronization of the multilayer network which can be appreciated in Fig 4 h) where we plot the mean phase for oscillators in both layers. The time series of the oscillators 1 and 15 (Fig 4(i)) show the amplification described in Eq 3. We can appreciate the synchronization with amplification of the multilayer network in the Fig $4(j, k, 1)$ where complete synchronization of some chosen oscillators is shown.

To appreciate the dynamics at the border of the separation of the domain D3 and D4 of the Fig 3(e) we present in Fig 5 (a) the variation of the phase of all oscillators of the slave layer for $C_{2}=2, \varepsilon_{1}=0.065721, \varepsilon_{2}=10 \varepsilon_{1}$ and interlayer coupling $\left(C_{0}\right)$ varying from 17.8 to 18.2 . At this range of values of the interlayer coupling, the slave layer presents different dynamics. Before $C_{0}=18$ this slave layer shows a synchronization of all oscillators of the slave layer. After this synchronisation there follows a slight zone of disturbance (the zoom is given at Fig 5 (b)) before the division into two groups which drives the layer towards the divergence. This abrupt change presented reminds of an explosive desynchronization 11 . Given the form of the connection (unidirectionnal coupling) between the first and second layer, the oscillators of the slave layer can only remain synchronous and stable for a certain range of values of $C_{0}$. This dynamic leads the slave layer to the divergence that we observe in Fig 5 (a) on the mean phase and in Fig 5 (c) with the order parameter. To understand more clearly the dynamics of the slave layer at this value of interlayer coupling where we have destruction of the synchronization, we show in Fig $5(\mathrm{~d}, \mathrm{e})$ the mean phase of the multilayer network and the correlation between the oscillators of the slave layer respectively. The
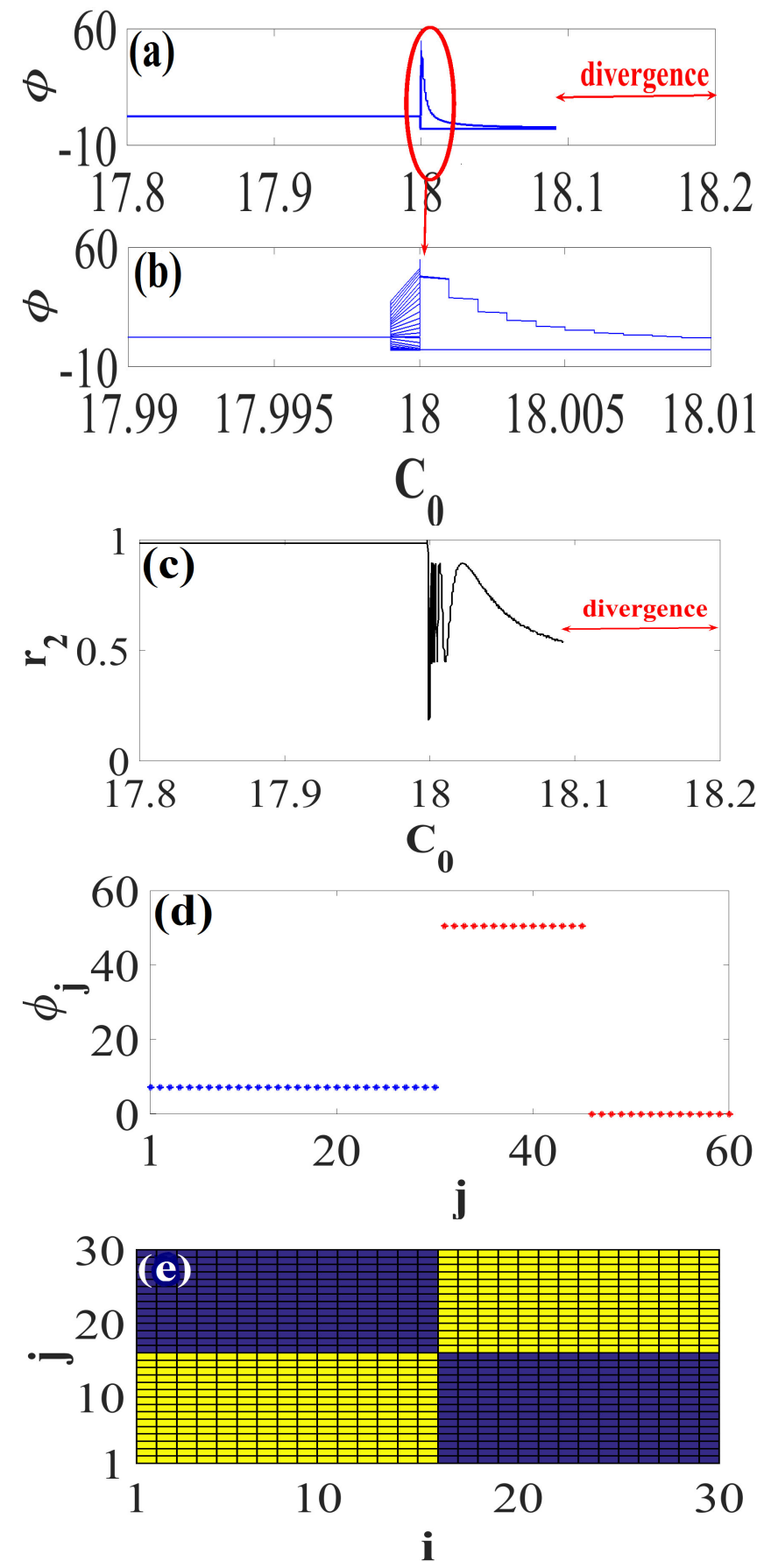

FIG. 5: Road to divergence: (a) Variation of the phases of the slave layer for $C_{2}=2, \varepsilon_{1}=0.065721$ and $\varepsilon_{2}=10 \varepsilon_{1}$. (b) Zoom of the variation of the phases of the slave layer for $C_{2}=2, \varepsilon_{1}=0.065721$ and $\varepsilon_{2}=10 \varepsilon_{1}$. (c)) Order parameter of the slave layer for $C_{2}=2, \varepsilon_{1}=0.065721$ and $\varepsilon_{2}=10 \varepsilon_{1}$. (d) Mean phase of the oscillators of the network and (e) correlation between the oscillators of the slave layer for $\varepsilon_{1}=0.065721$ and $\varepsilon_{2}=10 \varepsilon_{1}, C_{2}=2$ and $C_{0}=18$. (The yellow colour indicates the oscillators that synchronize and the blue colour those that do not synchronize).

mean phase presents a phase synchronization of all the oscillators of the first layer but in the slave layer we have two clusters formations. This cluster formation in the slave layer is confirmed using the correlation between the oscillators of 
the slave layer and then we can appreciate the formation of the these two clusters by the yellow colour.

\section{APPLICATIONS TO OTHER SYSTEMS}

The behavior of a multilayer network shown in the previous Sections is not restricted to a system of Rössler oscillators, it can also be obtained with other systems and topologies. In this Section we shall study a network of jerk oscillators and another of Liénard oscillators with different topologies and show that they reproduce the same behaviors.

\section{A. Synchronization with amplification in a multilayer network of jerk oscillators}

Here we investigate the dynamics of a multilayer network of jerk ${ }^{1}$ chaotic oscillators where the first layer is described by $\mathrm{Eq} 5$

$$
\left\{\begin{array}{l}
\dot{x}_{i}^{1}=I\left(x_{i}^{2}\right)+\varepsilon_{1}\left(x_{i+1}^{1}+x_{i-1}^{1}-2 x_{i}^{1}\right), \\
\dot{x}_{i}^{3}=\alpha\left(-x_{i}^{3}+I\left(x_{i}^{2}\right)\right) \\
\dot{x}_{i}^{3}=\beta\left(-x_{i}^{2}+x_{i}^{2}-\gamma x_{i}^{3}\right) .
\end{array}\right.
$$

The slave layer is described by Eq 6

$$
\left\{\begin{array}{l}
\dot{y}_{j}^{1}=\frac{I\left(x_{j}^{2}\right)}{C_{2}}+\varepsilon_{2}\left(y_{j+1}^{1}+y_{j-1}^{1}-2 y_{j}^{1}\right)+C_{0}\left(x_{j}^{1}-C_{2} y_{j}^{1}\right), \\
\dot{y}_{j}^{2}=\alpha\left(-y_{j}^{3}+I\left(x_{j}^{2}\right) / C_{2}\right), \\
\dot{y}_{j}^{3}=\beta\left(-y_{j}^{1}+y_{j}^{2}-\gamma y_{j}^{3}\right) .
\end{array}\right.
$$

Where the piecewise linear function is

$$
I\left(x^{2}\right)=\left\{\begin{array}{cl}
-x^{2} & \text { if } x^{2} \leq 1 \\
-1 & \text { otherwise }
\end{array}\right.
$$

$\alpha=0.025, \beta=0.765, \gamma=0.0938$ are the systems parameter. $\varepsilon_{1}$ and $\varepsilon_{2}$ are the intralayer coupling strength of the drive and slave layer of the network. The connection between the nodes of the same layer is bidirectional and they are arranged on a ring. Moreover, connection between the nodes of different layers is unidirectional and it only concerns the oscillators with the same index in both layers.

Eq 5 and Eq 6 were solved numerically considering $N=30$ jerk oscillators per layer with the systems parameter defined above. Fig 6 is obtained for $\varepsilon_{1}=2, \varepsilon_{2}=2$ and $C_{0}=2$. Two values for the amplification coefficient are considered: $C_{2}=0.5$ and $C_{2}=2$. For $C_{2}=0.5$ we can clearly appreciate in

\footnotetext{
${ }^{1}$ Based on Ref. ${ }^{27}$, a system is considered as jerk if the flow can be rewritten as a third order differential equation in a single scalar variable. For an isolated system, the jerk system can be defined as: $\dddot{x}_{2}=$ $\alpha\left(-\beta\left(-\dot{x}_{1}+\dot{x}_{2}-\gamma \ddot{x}_{1}+\frac{\gamma}{\alpha} \ddot{x}_{2}\right)+\dddot{x}_{1}\right)$. where $\left.\ddot{x}_{1}=I^{\prime}\left(x_{2}\right)\right) \dot{x}_{2}$ and $\dddot{x}_{1}=$ $\left.\left.I^{\prime \prime}\left(x_{2}\right)\right) \dot{x}_{2}^{2}+I^{\prime}\left(x_{2}\right)\right) \ddot{x}_{2}$. Then, $\dddot{x}_{1}$ is called jerk function.
}
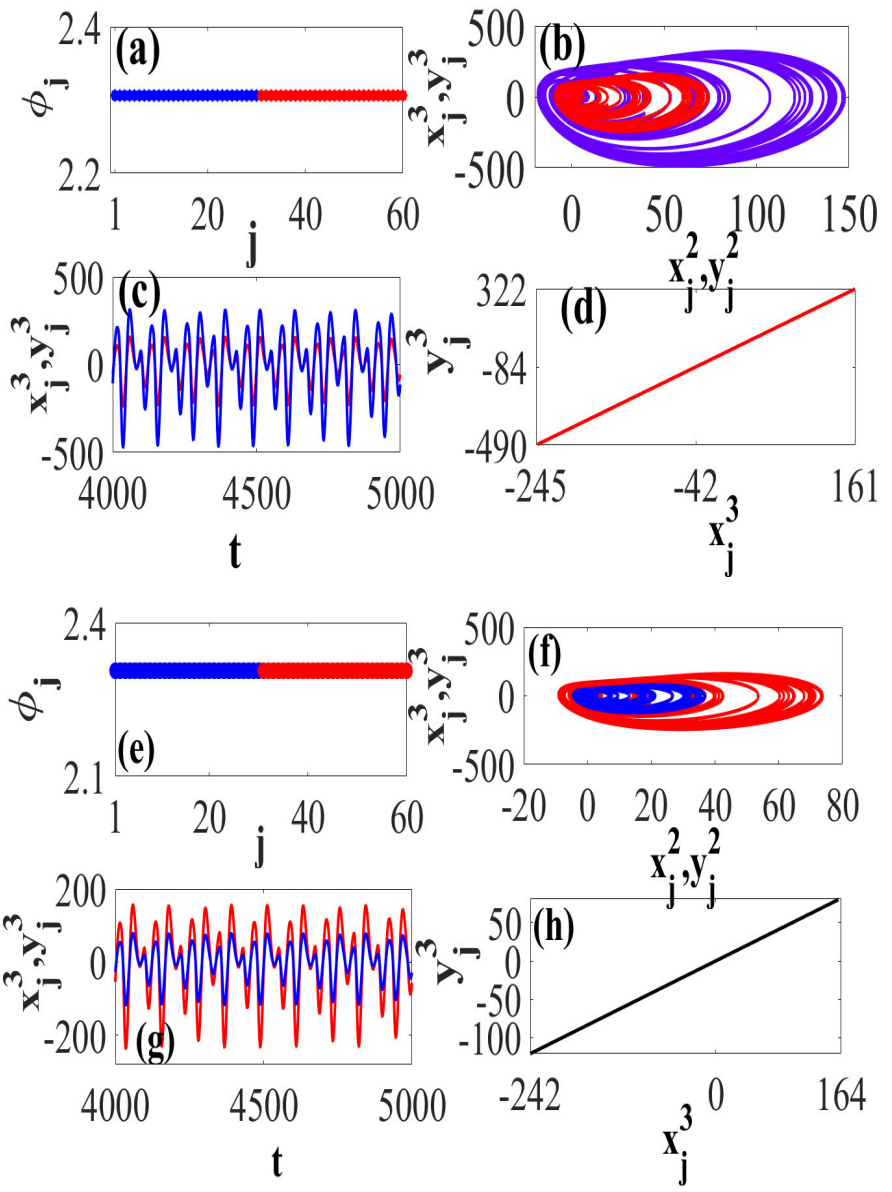

FIG. 6: Dynamics of the two layers of the network of jerk oscillators, where the master layer is represented in red and the slave layer in blue. For $\varepsilon_{1}=\varepsilon_{2}=2, C_{0}=5$ and $C_{2}=0.5$ : (a) mean phase of the first and second layer, (b) attractors of the oscillators in the master and slave layer, (c) time series of the oscillators of the master and slave layer and (d) synchronization between the oscillators of the first and second layer in the network. For $\varepsilon_{1}=\varepsilon_{2}=2, C_{0}=5$ and $C_{2}=2$ : (e) mean phase of the first and second layer, (f) attractors of the oscillators in the master and slave layer, (g) time series of the oscillators of the first and second layer and (h) synchronization between the oscillators of the first and second layer in the network.

Fig 6 a) the complete phase synchronization of all oscillators in both layers. This phase synchronization is a major condition to obtain amplification in the systems of the network. As defined by our model, it emerges that for this values of $C_{2}$ the oscillators of the slave network are supposed to be amplified compared to oscillators of the master layer (see Fig 6, b,c) where in red we show the oscillators of the master layer and in blue those of the slave layer). The amplification between master and slave layer is perfectly observed in Fig 6(d). In the second case we consider $C_{2}=2$. As in the previous case we have phase synchronization between the oscillators of both layers (see Fig 6.e)). For this value we have an amplification in the oscillators of the master layer (see Fig 6(f,g)). In the same vein we present in Fig 6(h) the synchronization between the oscillators in the first and second layer of the network.

Therefore, interlayer synchronization can be obtained with amplification or reduction depending on the value of the coef- 
ficient $C_{2}$.

B. Chimera states with amplification in a multilayer network of Liénard system

Let us consider a network of Liénard systems expressed as in reference ${ }^{15}$ where the authors chose to investigate the dynamics of the oscillators basing themselves on an attractive and repulsive global coupling. In the same vein, in the Refs. ${ }^{4243}$ the authors present some behaviour such as clusters, pattern formation, synchronization and so on according to the attractive and repulsive coupling.

In this subsection, we consider the Liénard model with a intralayer topology defined as in 15 .

First or master layer

$$
\left\{\begin{array}{l}
\dot{x}_{i}^{1}=x_{i}^{2}, \\
\dot{x}_{i}^{2}=-\alpha x_{i}^{1} x_{i}^{2}-\beta\left(x_{i}^{1}\right)^{3}-\gamma x_{i}^{1}+K\left[\left(\bar{x}^{2}-x_{i}^{2}\right)+\varepsilon_{1}\left(\bar{x}^{1}-x_{i}^{1}\right)\right] .
\end{array}\right.
$$

Where $\bar{x}^{1}=\frac{1}{N} \sum_{i=1}^{N} x_{i}^{1}$ and $\bar{x}^{2}=\frac{1}{N} \sum_{i=1}^{N} x_{i}^{2}$.

Second or slave layer

$$
\left\{\begin{array}{l}
\dot{y}_{j}^{1}=y_{j}^{2}+C_{0}\left(x_{i}^{1}-C_{2} y_{j}^{1}\right), \\
\dot{y}_{j}^{2}=-\alpha \frac{x_{j}^{1} x_{j}^{2}}{C_{2}}-\beta \frac{\left(x_{i}^{1}\right)^{3}}{C_{2}}-\gamma y_{j}^{1}+K\left[\left(\bar{y}^{2}-y_{j}^{2}\right)+\varepsilon_{2}\left(\bar{y}^{1}-y_{j}^{1}\right)\right] \\
+C_{0}\left(x_{j}^{2}-C_{2} y_{j}^{2}\right) .
\end{array}\right.
$$

With $\bar{y}^{1}=\frac{1}{N} \sum_{i=1}^{N} y_{i}^{1}$ and $\bar{y}^{2}=\frac{1}{N} \sum_{i=1}^{N} y_{i}^{2}$.

The system's parameters $\alpha, \beta$ and $\gamma$ are selected exactly as in $\frac{15}{15}, K$ is the strength of coupling, $\varepsilon_{1}$ and $\varepsilon_{2}$ are the intralayer global mean field coupling and $C_{2}$ is the amplification coefficient. The coupling between the nodes of different layers is unidirectional and it only concerns the oscillators with the same index in both layers and $N=100$ Liénard systems.

For the numerical simulation, we consider two cases, one where the amplifcation is less than one (the systems of the slave layer are amplified and the systems of master layer are reduced) and another when the amplification is greather than one (the systems of the slave layer are reduced and the systems of master layer are amplified).

Fig. 7 elaborates the Chimera-I states based on Refs! 15 |16. Fig. 7 (a) presents a snapshot of all the oscillators of both layers of the network (the first 100 systems correspond to the master layer and the rest is for slave layer). This figure presents a multichimera state in both master and slave layer (see Fig. 7 (c,d)) and a phase synchronization of both layers for $K=0.9, \varepsilon_{1}=\varepsilon_{2}=-0.57$, and $C_{2}=0.5$. For the same parameters we show in Fig 7 b) the attractor of the system for oscillator $j=1$ in the synchronization state and the attractor for $j=30$ in the incoherent state for both layers of the network (attractor red corresponds to the master layer and attractor blue for the slave layer). At $C_{2}=0.5$ the systems of the
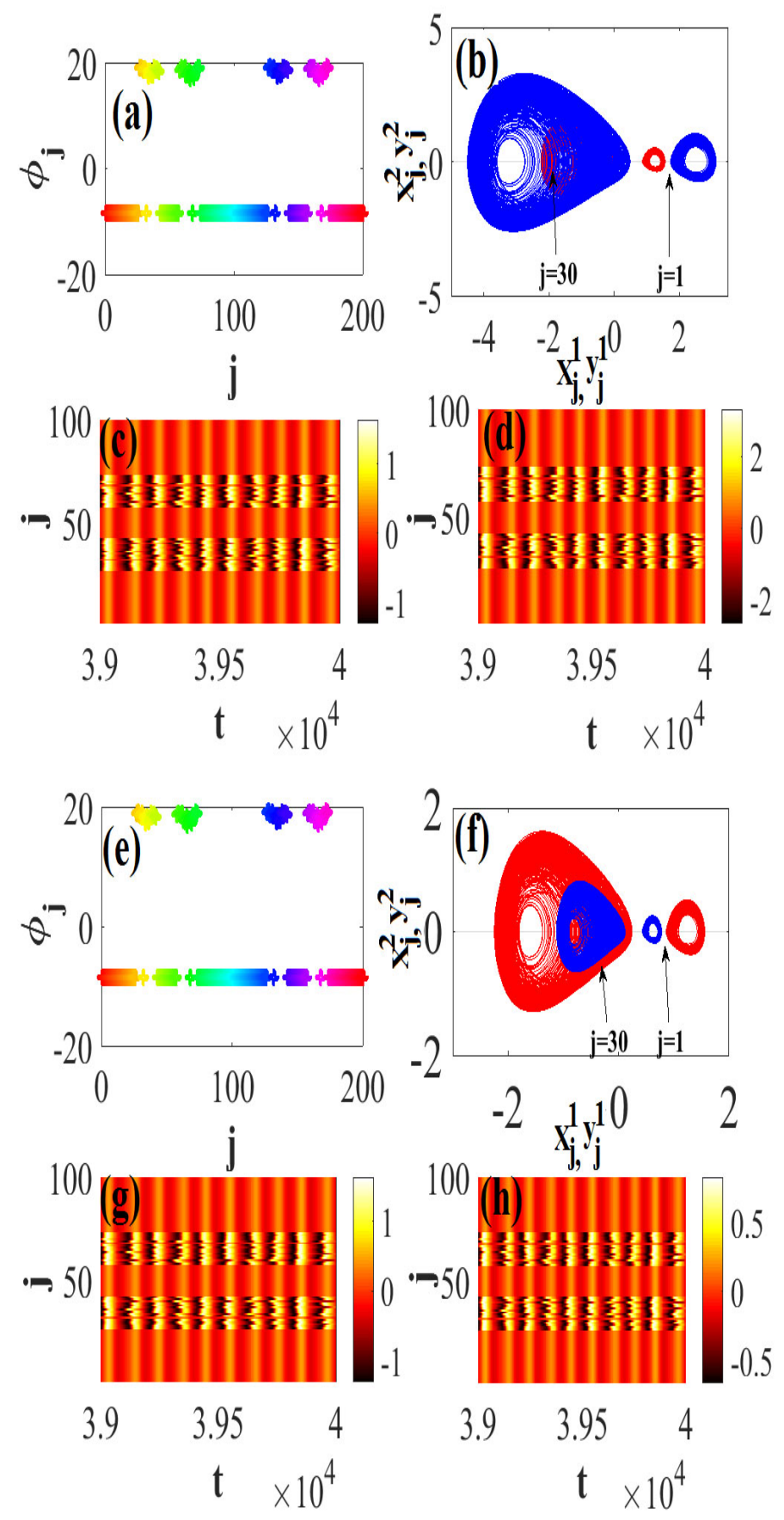

FIG. 7: Examples of behaviour of the multilayer network of Liénard systems. Amplification for $K=0.9, \varepsilon_{1}=\varepsilon_{2}=-0.57, C_{2}=0.5$ : (a) Snapshot of all the oscillators of the multilayer network showing a multichimera state, (b) phase portrait of the oscillators $j=1$ and $j=30$ of both layers and (c,d) temporal dynamics of all the nodes in the first and second layer in the network. Reduction for $K=0.9, \varepsilon_{1}=\varepsilon_{2}=-0.57$, $C_{2}=2$ : (e) Snapshot of all the oscillators of the multilayer network showing a multichimera state, (f) phase portrait of the oscillators $j=1$ and $j=30$ of both layers and $(\mathrm{g}, \mathrm{h})$ temporal dynamics of all the nodes in the first and second layer in the network. 
slave layer are supposed to be amplified as in Fig 7,b). In the same vein we present in Fig 7 (e,f,g,h) for $C_{2}=2$ the same behaviours as in Fig 7 (a,b,c,d). We observe the same behaviours except that are the systems of the master layer which are amplified as $X=2 Y$.

Based on these results, we conclude that this form of coupling can lead to a chimera or multichimera state with amplification or reduction depending on the value of the amplification coeficient.

Let us now consider the following parameters $K=1.5$ $\varepsilon_{1}=\varepsilon_{2}=-0.1$ that lead to a cluster formation and we choose two values of amplification parameter: (a) $C_{2}=0.5$ (see Fig $8(\mathrm{a}, \mathrm{b}, \mathrm{c}, \mathrm{d}))$ and (b) $C_{2}=2$ (see Fig $7(\mathrm{e}, \mathrm{f}, \mathrm{g}, \mathrm{h})$ ). According to these two cases we notice that this cluster state can be maintained with an amplification or reduction based on the value of $C_{2}$.

\section{CONCLUSION}

Summarizing, we have studied and characterized numerically the synchronization and the amplification of signals in a multilayer network of Rössler, jerk or Liénard oscillators. Using tools for studying synchronization in the network such as master stability function, the order parameter, we have demonstrated that, the existence of synchronization in the second layer is conditioned by the first. To obtain synchronization of both layers at the same value of the intralayer coupling, the amplification coefficient must be sufficiently low (see Fig 2 b)). The key role of amplification is demonstrated by analyzing the order parameter of the first and second layer. This parameter leads the network to different dynamics such as cluster formation and synchronization.

From a theoretical point of view, this work contributes to the advancement in the understanding of the phenomenon of synchronization and amplification of signals between two coupled networks. From a practical point of view, the results may be useful in many technological applications. For example, in several mechanical systems, the transmission of movements or orders is done through a driving belt or gear ${ }^{44}$. Concerning this process, one of the most important parameters is the transmission ratio, here represented by $C_{2}$, the amplification parameter. The amplification coefficient could be the transmission ratio between gears or pulley-belt systems $\frac{45}{\text {. }}$.

\section{DATA AVAILABILITY STATEMENT}

The data that supports the findings of this study are available within this article.

\section{ACKNOWLEDGEMENTS}

HAC thanks ICTP-SAIFR and FAPESP grant 2016/013437 for partial support. VC and FFF acknowledge that this study was financed in part by the Coordenação de Aperfeiçoamento
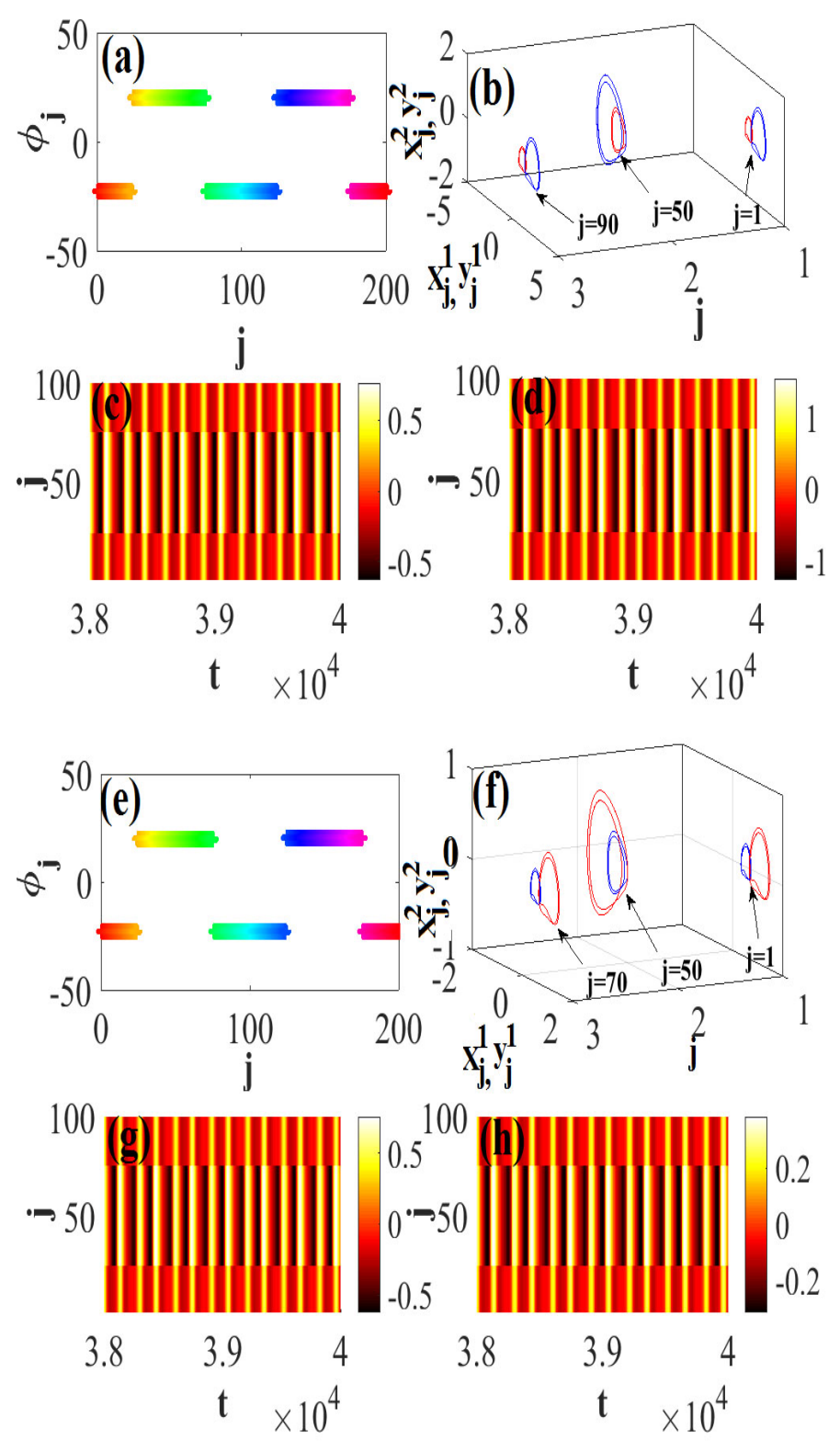

FIG. 8: Examples of behaviour of the multilayer network of Liénard systems. Amplification for $K=1.5, \varepsilon_{1}=\varepsilon_{2}=-0.1, C_{2}=0.5$ : (a) Snapshot of all the oscillators of the multilayer network showing a cluster, (b) phase portrait of the oscillators $j=1, j=50$ and $j=90$ of both layers and (c,d) temporal dynamics of all the nodes in the first and second layer in the network. Reduction for $K=1.5, \varepsilon_{1}=\varepsilon_{2}=-0.17, C_{2}=2$ : (e) Snapshot of all the oscillators of the multilayer network showing a cluster, (f) phase portrait of the oscillators $j=1, j=50$ and $j=70$ of both layers and (g,h)

temporal dynamics of all the nodes in the first and second layer in the network. 001 . 


\section{Appendix A}

\section{Synchronization conditions}

We consider $X\left(x_{i}^{1}, x_{i}^{2}, x_{i}^{3}\right)$ and $Y\left(y_{i}^{1}, y_{i}^{2}, y_{i}^{3}\right)$ the state vector of the $i^{t h}$ oscillator of the first and second layer respectively. The dynamics of the first and second layers are given respectively by:

$$
\left\{\begin{array}{l}
\dot{x}_{i}^{1}=-x_{i}^{2}-x_{i}^{3}+\varepsilon_{1} \sum_{k=1}^{N} A_{i k}^{1}\left(x_{k}^{1}-x_{i}^{1}\right) \\
\dot{x}_{i}^{2}=x_{i}^{1}+a x_{i}^{2} \\
\dot{x}_{i}^{3}=b x_{i}^{1}+x_{i}^{3}\left(x_{i}^{1}-c\right)
\end{array}\right.
$$

and

$$
\left\{\begin{array}{l}
\dot{y}_{j}^{1}=-y_{j}^{2}-y_{j}^{3}+\varepsilon_{2} \sum_{k=1}^{N} A_{j k}^{2}\left(y_{k}^{1}-y_{j}^{1}\right)+C_{0}\left(x_{j}^{1}-C_{2} y_{j}^{1}\right), \\
\dot{y}_{j}^{2}=\frac{x_{j}^{1}+a x_{j}^{2}}{C_{2}} \\
\dot{y}_{j}^{3}=b y_{j}^{1}+\frac{x_{j}^{3} x_{j}^{1}}{C_{2}}-c y_{j}^{3} .
\end{array}\right.
$$

Without any intralayer coupling $\left(\varepsilon_{1}=\varepsilon_{2}=0\right)$ both layers could synchronize with amplification depending on $C_{2}$.

We consider the synchronization error $e=X-C_{2} Y$ with $\varepsilon_{1}=$ $\varepsilon_{2}=0$. So, the error dynamical system which is obtained only for the couple of systems with the same index in the first and second layers is given by $\mathrm{Eq} \mathrm{A} 3$.

$$
\left\{\begin{array}{l}
\dot{e}_{i}^{1}=-e_{i}^{2}-e_{i}^{3}-C_{2} C_{0} e_{i}^{1}, \\
\dot{e}_{i}^{2}=0 \\
\dot{e}_{i}^{3}=b e_{i}^{1}-c e_{i}^{3} .
\end{array}\right.
$$

To simplify the demonstration, we consider the Lyapunov functions $v_{i}(i=1,2, \ldots, N)$ of system pairs $i$ and $j($ with $i=j$ ) of the first and second layer described as follow:

$$
v_{i}=\frac{1}{2}\left(\left(e_{i}^{1}\right)^{2}+\left(e_{i}^{2}\right)^{2}+\frac{1}{b}\left(e_{i}^{3}\right)^{2}\right)
$$

For any couple $(X, Y)$ we choose the following Lyapunov function candidate:

$$
v=\frac{1}{2}\left(\left(e^{1}\right)^{2}+\left(e^{2}\right)^{2}+\frac{1}{b}\left(e^{3}\right)^{2}\right) .
$$

It is established (assuming $a>0, b>0$ and $c>0$ ) that the system defined by Eq A3 is practically stable since the time derivative of the Lyapunov function in Eq $\mathrm{A} 5$ is bounded by a positive constant. This also means that the error between the driver and response systems is sufficiently small, but different from zero and could be considered as a tolerance in the synchronization condition 4 .

$$
\dot{v} \leq \frac{\left(e^{2}\right)^{2}}{4 c} .
$$

For the whole network, the Lyapunov function candidate $V$ can be defined as a sum of $v_{i}$ :

$$
V=\sum_{i=1}^{N} v_{i}
$$

And then for the whole network we can have:

$$
\dot{V} \leq \sum_{i=1}^{N} \frac{\left(e_{i}^{2}\right)^{2}}{4 c}
$$

Based on $\mathrm{Eq} \mathrm{A} 3$, this boundedness is ensured by the fact that $e_{i}^{2}(t)$ is constant due to the fact that $\dot{e}_{i}^{2}(t)=0$. Thereby, from Eq $\mathrm{A} 8 \mathrm{e} \rightarrow 0, X-C_{2} Y=0$ and induce $X=C_{2} Y$. we can obtain amplification or reduction depending on the value of the coefficient $C_{2}$.

\section{Appendix B}

\section{Preliminaries to the Investigation of the Dynamics of the Network: the Master Stability Function}

A regular problem that arises when analyzing the dynamics of a network is to find conditions that guarantee the synchronization of a system of coupled identical nonlinear oscillators, so that all the oscillators converge asymptotically towards the same state.

The Master Stability Function (MSF) developed by Pecora and Carroll ${ }^{20}$, constitutes one of the most useful tools to analyze the synchronization stability of a system of coupled identical nonlinear oscillators 120147. We develop here only some points of the principal idea of this method.

Considering a network of $N$ identical coupled chaotic oscillators (or nodes), let $\mathbf{x}_{i}$ a vector with $m$ components necessary to describe the state of the $i^{t h}$ node. In general, in absence of any interaction between the nodes of the network, the evolution of a node is given by Eq B1.

$$
\dot{\mathbf{x}}_{i}=\mathbf{F}\left(\mathbf{x}_{i}\right),
$$

In this Eq B1, $\mathbf{F}$ is a function defined from $\mathbb{R}^{m}$ to $\mathbb{R}^{m}$ and is used to define the local dynamics of the oscillators. To describe how the oscillators evolve when they are connected in a network, we need to consider not only the local dynamics presented at Eq B1, but also how each node is affected by the ones to which it is connected. So, the law governing the dynamical interaction of the $i^{t h}$ node is defined as:

$$
\dot{\mathbf{x}}_{i}=\mathbf{F}\left(\mathbf{x}_{i}\right)+\sigma \sum_{j=1}^{N} \mathbf{G}_{i j} \mathbf{H}\left(\mathbf{x}_{j}\right),
$$

where $\sigma$ is a coupling strength, $H: \mathbb{R}^{m} \longrightarrow \mathbb{R}^{m}$ is an arbitrary output function of each node's variables using in the coupling. If we put the network in a synchronized state, we have $\mathbf{x}_{i}=\mathbf{s}$ for all nodes, where $\mathbf{s}$ is any $\mathrm{m}$-dimensional vector. The only way all nodes have the same behavior is to have the sum, $\sum_{j=1}^{N} \mathbf{G}_{i j}$, be the same for all $i$. So, to obtain complete or identical synchronization the row sums of the coupling matrix must be the same for all rows. According to Pecora and Carrol ${ }^{20}$, we can collect the node dynamical variables, functions and coupling in:

$$
\begin{aligned}
\mathbf{x} & =\left[\mathbf{x}_{1}, \mathbf{x}_{2}, \ldots, \mathbf{x}_{N}\right], \\
\mathbf{F}(\mathbf{x}) & =\left[\mathbf{F}\left(\mathbf{x}_{1}\right), \mathbf{F}\left(\mathbf{x}_{2}\right), \ldots, \mathbf{F}\left(\mathbf{x}_{N}\right)\right] \\
\mathbf{H}(\mathbf{x}) & =\left[\mathbf{H}\left(\mathbf{x}_{1}\right), \mathbf{H}\left(\mathbf{x}_{2}\right), \ldots, \mathbf{H}\left(\mathbf{x}_{N}\right)\right],
\end{aligned}
$$


and $G$ be the matrix coupling coefficients $G_{i j}$, then based on $\mathrm{Eq} \mathrm{B} 3(\mathrm{a}, \mathrm{b}$ and $\mathrm{c}), \mathrm{Eq} \mathrm{B} 2$ can be written in a compact form as follow:

$$
\dot{\mathbf{x}}=\mathbf{F}(\mathbf{x})+\sigma \mathbf{G} \otimes \mathbf{H}(\mathbf{x}),
$$

where $\otimes$ is the Kronecker product. So, according to the form of $\mathrm{Eq} \mathrm{B} 2$, we can rewrite $\mathrm{Eq} 2$ in the same form with:

$$
\mathbf{F}\left(\mathbf{x}_{i}\right)\left\{\begin{array}{l}
\dot{x}_{i}^{1}=-x_{i}^{2}-x_{i}^{3}, \\
\dot{x}_{i}^{2}=x_{i}^{1}+a x_{i}^{2} \\
\dot{x}_{i}^{3}=b x_{i}^{1}+x_{i}^{3}\left(x_{i}^{1}-c\right) .
\end{array}\right.
$$

According to ${ }^{20}$, for an all-to-all coupling scheme, the connectivity matrix can be defined as in matrix $G$. To couple the nodes of the layer we choose the $\mathbf{x}^{1}$ component and then the matrix $H$ can be defined as in $\mathrm{Eq} \mathrm{B} 6$

$\mathbf{H}=\left(\begin{array}{lll}1 & 0 & 0 \\ 0 & 0 & 0 \\ 0 & 0 & 0\end{array}\right), \mathbf{G}=\left(\begin{array}{cccccc}1-N & 1 & \cdots & 1 & 1 & 1 \\ 1 & 1-N & \cdots & 1 & 1 & 1 \\ \vdots & \vdots & \cdots & \vdots & \vdots & \vdots \\ 1 & 1 & \cdots & 1 & 1-N & 1 \\ 1 & 1 & \cdots & 1 & 1 & 1-N\end{array}\right)$,

The Master Stability Function studies the stability of the global synchronization in the network. Therefore, the synchronous state is obtained when $\mathbf{x}_{1}=\mathbf{x}_{2}=\ldots=\mathbf{x}_{N}=\mathbf{s}$.

Suppose our system is synchronized and we perturb it so that each node is, in general, slightly "away" from the synchronized motion. Let us consider $\xi_{i}$ a small perturbation of the $i^{t h}$ node of the network. so that after the perturbation $\mathbf{x}_{i}=\mathbf{s}+\xi_{i}$. For $\mathrm{N}$ oscillators of the first layer the collections of the variations can be expressed as $\xi=\left(\xi_{1}, \xi_{2}, \ldots, \xi_{N}\right)$. Now we can derive an equation of motion for the small perturbations that we will use to explore if the synchronized state is unstable or stable. So, replacing the perturbation $\mathbf{x}_{i}=\mathbf{s}+\xi_{i}$ in $\mathrm{Eq} \mathrm{B} 2$ and using Taylor theorem expand of $\mathbf{F}\left(\mathbf{s}+\xi_{i}\right)$ and $\mathbf{H}\left(\mathbf{s}+\xi_{i}\right)$ to first order (since $\xi_{i}$ is small) we have the following variational equation:

$$
\dot{\xi}_{i}=\mathbf{D F}(\mathbf{s}) \xi_{i}+\sigma \sum_{j=1}^{N} \mathbf{G}_{i j} \mathbf{D H}(\mathbf{s}) \xi_{j},
$$

Using tensor notation, we can write $\mathrm{Eq} \mathrm{B} 7$ in a more compact form:

$$
\dot{\xi}=\left[\mathbf{1}_{\mathbf{N}} \otimes \mathbf{D F}(\mathbf{s})+\sigma \mathbf{G} \otimes \mathbf{D H}(\mathbf{s})\right] \xi,
$$

where $\mathbf{1}_{\mathbf{N}}$ is the identity matrix of order $N, \mathbf{D F}$ and $\mathbf{D H}$ are the $N \times N$ Jacobian matrices of the corresponding vector functions.

The solution of Eq $\mathrm{B} 8$ can be in the form $\xi_{i} \sim \exp \mu_{i} t$. The exponents $\mu$ tell us if the perturbation grows $(\mu>0)$ or shrinks $(\mu<0)$, the former indicating a direction that is unstable and the latter a stable direction. After diagonalization of the second term of $\mathrm{Eq} \overline{\mathrm{B} 8}$ we obtain the variational equations which are diagonal in the node coordinates and are now uncoupled and individually given by:

$$
\dot{\xi}_{\mathbf{k}}=\left[\mathbf{D F}(\mathbf{s})+\sigma \alpha_{k} D \mathbf{H}(\mathbf{s})\right] \xi_{k} .
$$

where $\alpha_{k}$ is an eigenvalue of $\mathbf{G}, k=1,2, \ldots N$. For each $k$, the form of each block of the $\mathrm{Eq} \mathrm{B} 8$ does not change, only the scalar multiplier $\sigma \alpha_{k}$ differs for each block.

Therefore, these steps lead us to design the following master stability equation:

$$
\dot{\xi}=[\mathbf{D F}(\mathbf{s})+\sigma \alpha \mathbf{D H}(\mathbf{s})] \xi .
$$

Computing the Largest Lyapunov exponent of this Master Stability Equation Eq B10 we obtain what Pecora and Carroll called The Master Stability Function and therefore we achieve a stable synchronization state if the MSF turns negative $e^{20148 / 49}$.

\section{Appendix C}

\section{Calculation of the Order Parameter}

Collective behavior of such an N-oscillator system is conveniently described by the order parameter. The evaluation of this order parameter ${ }^{\sqrt{12}}$ used the phase of each oscillator of the network. To define the phase let us consider an arbitrary signal $s(\tau)$ with time $\tau$ and its Hilbert transformation to be $\tilde{s}(\tau)$, we have:

$$
\psi(\tau)=s(\tau)+i \tilde{s}(\tau)=R(\tau) \exp ^{i \phi(\tau)},
$$

where $R(\tau)$ is the amplitude and $\phi(\tau)$ the phase of the variable $s(\tau)$. If the instantaneous phase is $\phi_{i}(\tau)$, it can be determined through the following relation:

$$
\phi_{i}(\tau)=\tan ^{-1}\left[\frac{\tilde{s}_{i}(\tau)}{s_{i}(\tau)}\right] .
$$

In this paper the calculation of the phase was carried out using in each case the variable that best describes the dynamics of the system.

Thus, from the expression of the phase $\phi_{i}$, the mean phase $\phi$ is an algebraic average calculated on the $N$ oscillators of the layer. So, for a network of $\mathrm{N}$ oscillators the order parameter can be expressed as:

$$
r=\frac{1}{N} \sum_{i=1}^{N} e^{j \phi_{i}}
$$

where $j^{2}=-1$, when $r \rightarrow 1$, phase synchronization is reached and when $r \approx 0$, the network is desynchronized.

\section{REFERENCES}

${ }^{1}$ I. Leyva, I. Sendiña-Nadal, R. Sevilla-Escoboza, V. Vera-Avila, P. Chholak, and S. Boccaletti, "Relay synchronization in multiplex networks," Sci. Rep. 8, 1 (2018). 
${ }^{2}$ M. De Domenico, A. Solé-Ribalta, E. Cozzo, M. Kivelä, Y. Moreno, M. A. Porter, S. Gómez, and A. Arenas, "Mathematical formulation of multilayer networks," Phys. Rev. X 3, 041022 (2013).

${ }^{3}$ X. Zhang, S. Boccaletti, S. Guan, and Z. Liu, "Explosive synchronization in adaptive and multilayer networks," Phys. Rev. Lett. 114, 038701 (2015).

${ }^{4}$ S. Jalan, A. D. Kachhvah, and H. Jeong, "Explosive synchronization in multilayer dynamically dissimilar networks," Journal of Computational Science, 101177 (2020).

${ }^{5}$ I. Belykh, D. Carter, and R. Jeter, "Synchronization in multilayer networks: when good links go bad," SIAM Journal on Applied Dynamical Systems 18, 2267 (2019).

${ }^{6}$ K. A. Blaha, K. Huang, F. Della Rossa, L. Pecora, M. Hossein-Zadeh, and F. Sorrentino, "Cluster synchronization in multilayer networks: A fully analog experiment with $1 \mathrm{c}$ oscillators with physically dissimilar coupling," Phys. Rev. Lett. 122, 014101 (2019).

${ }^{7}$ A. Arenas, A. Díaz-Guilera, J. Kurths, Y. Moreno, and C. Zhou, "Synchronization in complex networks," Phys. Rep. 469, 93 (2008).

${ }^{8}$ C. del Genio, J. Gómez-Gardeñes, I. Bonamassa, and S. Boccaletti, "Synchronization in networks with multiple action layers," Sci. Adv. 2, e1601679 (2016).

${ }^{9}$ Y. Zhang, G. Hu, H. A. Cerdeira, S. Chen, T. Braun, and Y. Yao, "Partial synchronization and spontaneous spatial ordering in coupled chaotic systems," Phys. Rev. E 63, 026211 (2001).

${ }^{10} \mathrm{P}$. Ji, T. K. D. Peron, F. A. Rodrigues, and J. Kurths, "Analysis of cluster explosive synchronization in complex networks," Phys. Rev. E 90, 062810 (2014).

${ }^{11}$ R. Lauter, A. Mitra, and F. Marquardt, "From kardar-parisi-zhang scaling to explosive desynchronization in arrays of limit-cycle oscillators," Phys. Rev. E 96, 012220 (2017).

${ }^{12}$ Y. Kuramoto and D. Battogtokh, "Coexistence of coherence and incoherence in nonlocally coupled phase oscillators," Nonlinear Phenom. Complex Syst. 5, 380 (2002).

${ }^{13}$ L. Schmidt, K. Schönleber, K. Krischer, and V. García-Morales, "Coexistence of synchrony and incoherence in oscillatory media under nonlinear global coupling," Chaos 24, 013102 (2014).

${ }^{14}$ L. Schmidt and K. Krischer, "Clustering as a prerequisite for chimera states in globally coupled systems," Phys. Rev. Lett. 114, 034101 (2015).

${ }^{15}$ A. Mishra, C. Hens, M. Bose, P. K. Roy, and S. K. Dana, "Chimeralike states in a network of oscillators under attractive and repulsive global coupling," Phys. Rev. E 92, 062920 (2015).

${ }^{16}$ D. Dudkowski, Y. Maistrenko, and T. Kapitaniak, "Different types of chimera states: An interplay between spatial and dynamical chaos," Phys. Rev. E 90, 032920 (2014).

${ }^{17}$ C. Castellano, S. Fortunato, and V. Loreto, "Statistical physics of social dynamics," Rev. Mod. Phys. 81, 591 (2009).

${ }^{18}$ R. Pastor-Satorras, C. Castellano, P. Van Mieghem, and A. Vespignani, "Epidemic processes in complex networks," Rev. Mod. Phys. 87, 925 (2015).

${ }^{19}$ Y.-Y. Liu, J.-J. Slotine, and A.-L. Barabási, "Controllability of complex networks," nature 473, 167 (2011).

${ }^{20}$ L. M. Pecora and T. L. Carroll, "Master stability functions for synchronized coupled systems," Phys. Rev. Lett. 80, 2109 (1998).

${ }^{21}$ S. Boccaletti, G. Bianconi, R. Criado, C. I. Del Genio, J. Gómez-Gardenes, M. Romance, I. Sendina-Nadal, Z. Wang, and M. Zanin, "The structure and dynamics of multilayer networks," Phys. Rep. 544, 1 (2014).

${ }^{22}$ J. I. Mondragon, Raul J. and G. Bianconi, "Multilink communities of multiplex networks," PloS one 13, e0193821 (2018).

${ }^{23}$ S. Boccaletti, R. Criado, M. Romance, and J. J. Torres, "Introduction to focus issue: Complex dynamics in networks, multilayered structures and systems," Chaos 26, 065101 (2016).

${ }^{24}$ I. Leyva, R. Sevilla-Escoboza, I. Sendina-Nadal, R. Gutierrez, J. Buldu, and S. Boccaletti, "Inter-layer synchronization in nonidentical multi-layer networks,".

${ }^{25}$ S. Ghosh, A. Zakharova, and S. Jalan, "Non-identical multiplexing pro- motes chimera states," Chaos, Solitons \& Fractals 106, 56 (2018).

${ }^{26}$ O. E. Rössler, "Continuous chaos-four prototype equations," Annals of the New York Academy of Sciences 316, 376 (1979).

${ }^{27}$ P. Louodop, S. Saha, R. Tchitnga, P. Muruganandam, S. K. Dana, and H. A. Cerdeira, "Coherent motion of chaotic attractors," Phys. Rev. E 96, 042210 (2017).

${ }^{28}$ W. Zou, D. Senthilkumar, R. Nagao, I. Z. Kiss, Y. Tang, A. Koseska, J. Duan, and J. Kurths, "Restoration of rhythmicity in diffusively coupled dynamical networks," Nature communications 6, 1 (2015).

${ }^{29} \mathrm{D}$. Ghosh, T. Banerjee, and J. Kurths, "Revival of oscillation from meanfield-induced death: Theory and experiment," Physical Review E 92, 052908 (2015).

${ }^{30}$ R. Karnatak, R. Ramaswamy, and A. Prasad, "Amplitude death in the absence of time delays in identical coupled oscillators," Physical Review E 76, 035201 (2007).

${ }^{31}$ S. Cafieri, L. Cellier, F. Messine, and R. Omheni, "Combination of optimal control approaches for aircraft conflict avoidance via velocity regulation," Optimal Control Applications and Methods 39, 181 (2018).

${ }^{32}$ H.-B. Chen, Y.-T. Sun, J. Gao, C. Xu, and Z.-G. Zheng, "Order parameter analysis of synchronization transitions on star networks," Frontiers of Physics 12, 120504 (2017).

${ }^{33} \mathrm{H}$. Hong and M. Choi, "Phase synchronization and noise-induced resonance in systems of coupled oscillators," Physical Review E 62, 6462 (2000).

${ }^{34}$ G. Hu, Y. Zhang, H. A. Cerdeira, and S. Chen, "From low-dimensional synchronous chaos to high-dimensional desynchronous spatiotemporal chaos in coupled systems," Phys. Rev. Lett. 85, 3377 (2000).

${ }^{35}$ Y. Maistrenko, O. Popovych, O. Burylko, and P. A. Tass, "Mechanism of desynchronization in the finite-dimensional kuramoto model," Phys. Rev. lett. 93, 084102 (2004).

${ }^{36}$ C. Allefeld, M. Müller, and J. Kurths, "Eigenvalue decomposition as a generalized synchronization cluster analysis," International Journal of Bifurcation and Chaos 17, 3493 (2007).

${ }^{37}$ J. Singha, V. Tchuyang, P. Louodop, R. Tchitnga, H. A. Cerdeira, and N. Gupte, "Spatial splay states in coupled map lattices and josephson junction arrays," Indian Academy of Sciences 1, 3493 (2017).

${ }^{38} \mathrm{~S}$. Nichols and K. Wiesenfeld, "Ubiquitous neutral stability of splay-phase states," Phys. Rev. A 45, 8430 (1992).

${ }^{39} \mathrm{H}$. Fujisaka and T. Yamada, "Stability theory of synchronized motion in coupled-oscillator systems," Prog. Theor. Phys. 69, 32 (1983).

${ }^{40}$ L. M. Pecora and T. L. Carroll, "Synchronization in chaotic systems," Phys. Rev. Lett. 64, 821 (1990).

${ }^{41}$ K. Pearson, "Notes on the history of correlation," Biometrika 13, 25 (1920).

${ }^{42} \mathrm{H}$. Hong and S. H. Strogatz, "Mean-field behavior in coupled oscillators with attractive and repulsive interactions," Phys. Rev. E 85, 056210 (2012).

${ }^{43}$ K. Sathiyadevi, V. Chandrasekar, D. Senthilkumar, and M. Lakshmanan, "Distinct collective states due to trade-off between attractive and repulsive couplings," Phys. Rev. E 97, 032207 (2018).

${ }^{44}$ J. F. Denijs and M. Lindner, "Transmission for converting rotary motion into linear motion," (1997), uS Patent 5,690,567.

${ }^{45}$ A. Farshidianfar and A. Saghafi, "Identification and control of chaos in nonlinear gear dynamic systems using melnikov analysis," Physics Letters A 378, 3457-3463 (2014).

${ }^{46}$ F. M. Kakmeni, S. Bowong, D. Senthikumar, and J. Kurths, "Practical time-delay synchronization of a periodically modulated self-excited oscillators with uncertainties," Chaos: An Interdisciplinary Journal of Nonlinear Science 20, 043121 (2010).

${ }^{47}$ S. Majhi, M. Perc, and D. Ghosh, "Chimera states in uncoupled neurons induced by a multilayer structure," Sci. Rep. 6, 39033 (2016).

${ }^{48}$ L. Tang, X. Wu, J. Lü, J.-a. Lu, and R. M. D'Souza, “Master stability functions for complete, intralayer, and interlayer synchronization in multiplex networks of coupled rössler oscillators," Phys. Rev. E 99, 012304 (2019).

${ }^{49}$ L. Huang, Q. Chen, Y.-C. Lai, and L. M. Pecora, "Generic behavior of master-stability functions in coupled nonlinear dynamical systems," Phys. Rev. E 80, 036204 (2009). 